\title{
VHF Data Exchange System (VDES): an enabling technology for maritime communications
}

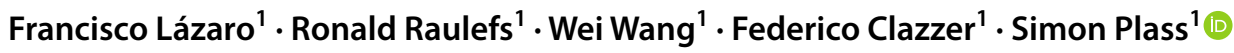

Received: 30 March 2017 / Revised: 4 July 2018 / Accepted: 5 July 2018 / Published online: 20 July 2018

(c) The Author(s) 2018

\begin{abstract}
This work presents an overview of the radio interface of VHF Data Exchange System (VDES), which is currently on its way to become an ITU standard supported by International Association of Marine Aids to Navigation and Lighthouse Authorities. VDES includes the already existing collision avoidance tracking system Automatic Identification System and the messaging system Application Specific Messages. Additionally, a new third component for digital maritime communications of any kind, named VHF Data Exchange is included. On the one hand, there is a traditional terrestrial component, on the other hand, a satellite communication link is also envisioned partly by the same spectrum usage. In this article, focus is given towards the technical design aspects and challenges of this hybrid communications transmission scheme.
\end{abstract}

Keywords VHF $\cdot$ Maritime digital communications $\cdot$ Satellite $\cdot$ Terrestrial $\cdot$ Coding and modulation $\cdot$ Random access

\section{Introduction}

In the globalized world in which we live, transportation is becoming more and more important. The number of passengers and products carried by transportation systems over land, air and sea increases every year, and is expected to keep growing over the next decades. This ever increasing traffic results in a number of challenges, some of which are related.

The research leading to these results has been carried out under the framework of the project "R\&D for the maritime safety and security and corresponding real time services". The project started in 2013 and is led by the Program Coordination Defence and Security Research within the German Aerospace Center (DLR).

Simon Plass

Simon.Plass@dlr.de

Francisco Lázaro

Francisco.LazaroBlasco@dlr.de

Ronald Raulefs

Ronald.Raulefs@dlr.de

Wei Wang

Wei.Wang@dlr.de

Federico Clazzer

Federico.Clazzer@dlr.de

1 German Aerospace Center (DLR), Institute of Communications and Navigation, Wessling, Germany
The first attempt towards the digitalization of maritime transportation was the introduction of Automatic Identification System (AIS) [1, 2]. The main driver behind the introduction of AIS was safety. More concretely, the main goal of AIS was enabling a type of broadcast communication in which vessels periodically report their position, course and speed, to avoid collisions. However, since the introduction of AIS, new application and services have arisen. Among others, AIS is nowadays also used for aids to navigation, search and rescue, and fleet monitoring. One of the functionalities of AIS which proved to be very useful was its support for Application Specific Messages (ASM) [3], which allow authorities to define custom AIS message types. Initially, only very large vessels (over 300 gross tonnages) were mandated to fit a Class A AIS transceiver. After some time a lower cost type of AIS transceiver was specified (Class B) [4]. This lower cost transceiver was mandated in some countries for smaller vessels, but the adoption of AIS has been such that in many cases vessels are equipped with AIS transceivers even though they are not mandated to do so. The growth in AIS has been such, that in some of the most crowded waters the system is as of today already overloaded.

Given the danger to AIS' main mission, collision avoidance, that this overload can represent, the International Association of Marine Aids to Navigation and Lighthouse Authorities (IALA) and a number of national maritime authorities started the work on the VHF Data Exchange 


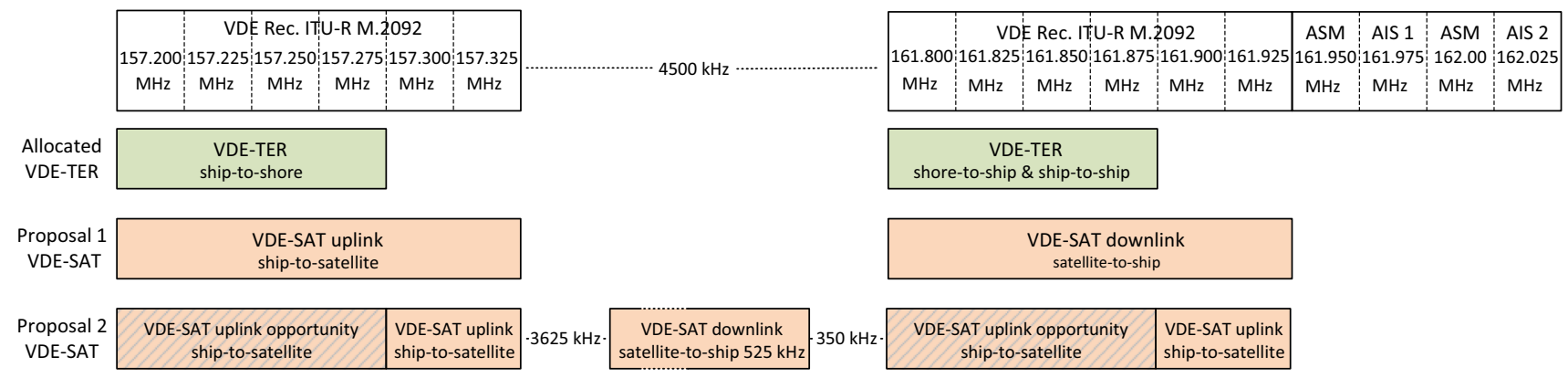

Fig. 1 The channel separation of AIS, ASM and VDE in the maritime VHF band

System (VDES) [6]. Rather than an evolution of AIS, VDES is a communications system encompassing different communications subsystems, one of them being AIS. A second subsystem of VDES are dedicated ASM channels, whose purpose is decreasing the load of AIS channels. Furthermore, VDES has a third subsystem, called VDE, which allows higher rate communications, and is highly flexible to be able to support a variety of services in the future. A key characteristic of VDES is that it does not only support direct ship to ship and ship to coast communication, but it also foresees a satellite component specifically for VDE.

VHF Data Exchange System can be a disrupting technology in the maritime sector, enabling the use of services such as Vessel Traffic Service (VTS) which are difficult to deploy nowadays due to the lack of a data link. But even more importantly, in the near future VDES will have the necessary flexibility to support novel services which we have not even thought of. However, there are also some challenges that VDES will face in the future. The first results from the world wide spectrum assignment of the International Telecommunication Union (ITU) at the world radio conference 2015 (WRC-15) [7]. For the terrestrial VDE part $100 \mathrm{kHz}$ spectrum were assigned for the down- and uplink as well as two $25 \mathrm{kHz}$ channels for ASM (terrestrial and satellite). The proposed spectrum band for the satellite was delayed until further studies about potential interference issues on land are investigated. The spectrum assignment for the satellite is now an agenda item at the next WRC in 2019. The second challenge VDES is facing is also in part regulatory. At the time of writing of this article, VDE is not foreseen to be made mandatory for vessels of any kind by International Maritime Organization (IMO) or other national authorities. Without a regulatory need to support VDE, manufacturers will have to provide low-cost entry terminals to VDES. In other words, in order for VDES to achieve a high penetration, it is necessary that fully VDES compliant receivers (including AIS, ASM and VDE) are available at a price similar to that of AIS only receivers.

The goal of this paper is providing a description of VDES highlighting its key features. Furthermore, we describe the status of the standardization process and the regulatory challenges that VDES is currently facing. Finally, we also list several research directions relevant for the future of VDES.

The remaining of the paper is structured as follows. The next section describes the different use cases relevant to VDE followed by a description of the architecture of VDES together with the principles of VDES operation. The subsequent section enters into some technical aspects of VDES, including channel modeling, modulation and coding and Medium Access (MAC) protocols followed by the current status of VDES standardization and the regulatory that lie ahead. Before the concluding section we focus on research directions relevant to the future of VDES. Finally, main conclusions are summarized.

\section{VDES system architecture}

The architecture of the VDES comprises three components. These are AIS,ASM and VDE. Figure 1 shows the two potential allocations for the different channels of the AIS, ASM and VDE systems.

The AIS system was developed in the 1990s and its original idea is to avoid collisions between vessels without shore authorities in reach [2]. AIS has the highest priority of all three systems because of the safety relevance. AIS transceivers exchange their current position and velocity information and tracking information. Within reach of shore authorities, especially in busy harbors, the vessel traffic services uses the AIS information to the International Maritime Organization demanded in 2002 for large vessels with more than 300 gross tonnage that AIS is mandatory equipment. Revised specifications in 2007 defined Class B AIS transceivers [4]. The less complex specifications enabled a new demand from local authorities to track vessels in their coastal waters for smaller ships to have a Class B transceiver. In the following years, e.g., in Europe new mandates for local vessels demanded AIS Class A transceivers also for other smaller types of commercial vessels. The AIS data is transmitted with different power levels that range from $1 \mathrm{~W}$ up to $12.5 \mathrm{~W}$ 




(a)

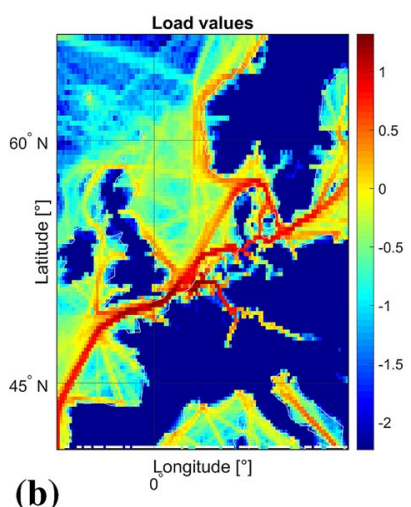

(b)

Fig. 2 Vessel traffic along Western Europe given by exactEarth ${ }^{\circledR}$. a Expected coverage for satellite VDE beyond the coverage of the coastal stations. b Expected traffic density overall
ASM was originally developed to reduce the load on the AIS channels for dedicated information [3]. However, AIS is designed to provide information about the whereabouts of the vessel and its heading to avoid collisions regularly depending on the velocity and movement changes. Therefore, the motivation of ASM is to offload the AIS channel with specific a-priori defined messages. The physical layer is similar to VDE to have common hardware implementations that can support either ASM or VDE. However, the ASM channels are next to the AIS channels and therefore, require a more strict spectrum mask which results in a less efficient channel usage.

Finally, VDE offers its frequency band to transmit any data. The VHF system is the second step after the introduction of AIS towards a fully digitized maritime VHF band. The ambition is to reuse main parts of the RF-hardware from

Fig. 3 VDE system concept and available communication links

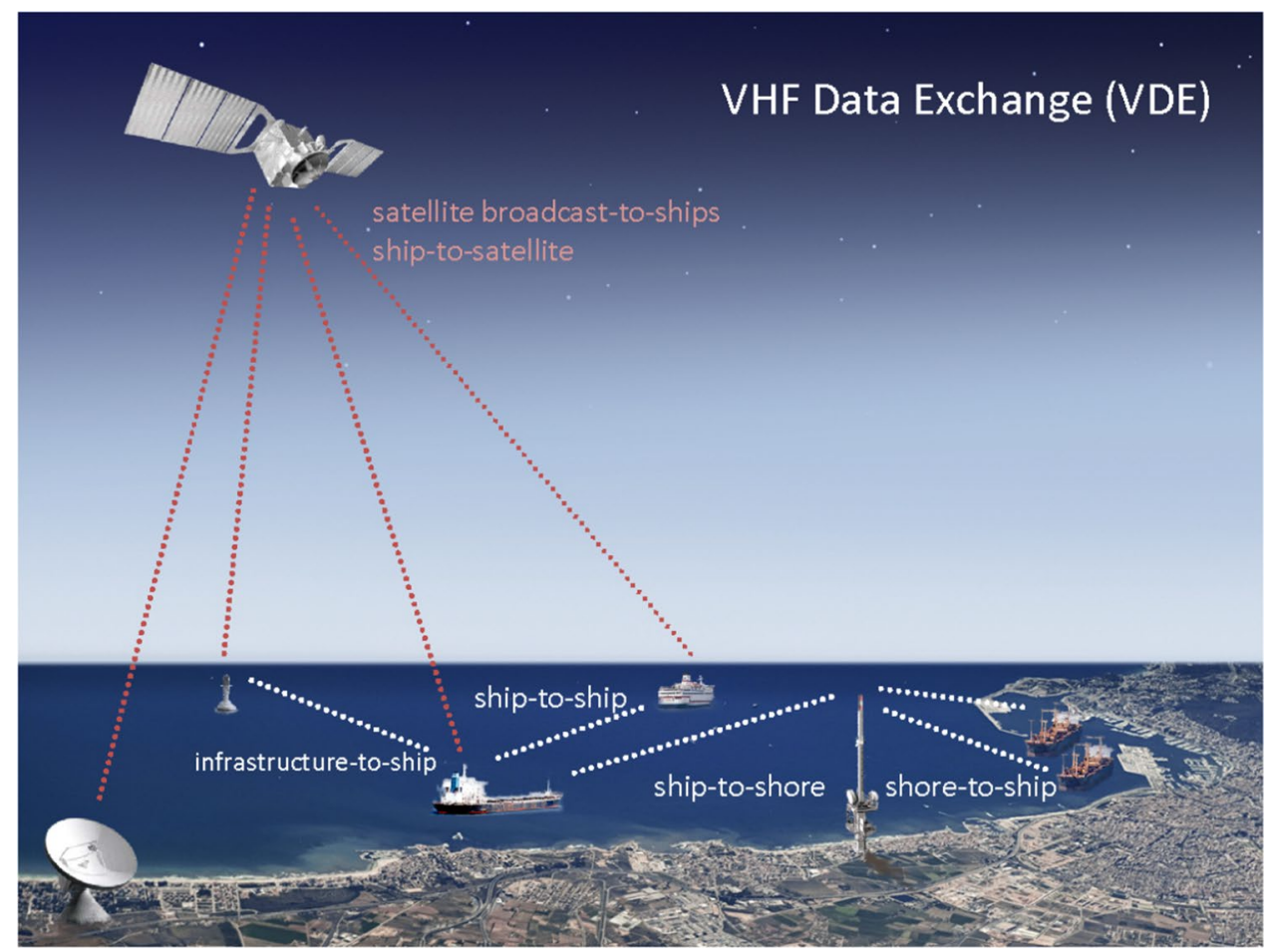

depending on which amplifiers are used. In space the AIS signals are received and collected in a parasitic manner since 2008. The key challenge is collecting and detecting AIS data in an area that is approximately 40 times larger than terrestrial coverage [8, 9]. However, AIS data is repeatedly broadcasted and the satellite receivers and its operators offer AIS data not as real-time service. Therefore, any post-processing could be done off-line and cope with the different interference levels of the different transmitters. The AIS system is also integrated into other maritime devices such as Aids to Navigation (AtoN), Search and Rescue Transmitter, and Man Over-Board units. the AIS components, such as the cabling and the antenna. Figure $2 \mathrm{a}$ shows the density of the vessels that are out of range of the coastal coverage (above $45 \mathrm{M}$ ). Figure $2 \mathrm{~b}$ includes the coastal coverage of AIS, and therefore, the potential data load of vessels from the shore. About $47.15 \%$ of the vessels are out of coverage of the coastal AIS transceiver and would rely on the connectivity to a satellite system (Fig. 3).

The coexistence of the terrestrial and the satellite system is an important aspect for VDE. For now two different approaches have been investigated. The first approach uses 
the terrestrial spectrum in the downlink in parallel also by the satellites in the downlink. The expected interference from the satellite signal on the terrestrial reception, which is limited to $1 \mathrm{~W}$ transmit power, is low. However, the satellite coverage is also affected by terrestrial shore stations, not only within terrestrial coverage area, but also beyond that point. The second approach separates the downlink channels of terrestrial and satellite in frequency and avoids any overlap. Within the large spectrum gap between the downlink and the uplink channels the satellite downlink channel now occupies up to $500 \mathrm{kHz}$. The uplink channels are split to potential slots next to the terrestrial down- and uplink channel.

A common design goal of the terrestrial and the satellite VDE system is to keep the development costs low and to target a simple replacement of an AIS transceiver with a single "VDES box". This is a challenge as compared to an AIS transceiver the goal is to support higher modulation schemes, such as 16-QAM which require a linear controlled amplifier with a significant back-off to avoid any distortions. Therefore, it is envisaged to reuse hardware parts, such as the cabling and the antenna. Further, the transceiver makes use of common system blocks, such as the modulation, coding and interleaving blocks for both VDE systems.

\section{VDE use cases}

The different use cases that VDE [6] addresses are the following:

- Search and Rescue (SAR) communications. This service allows ships to communicate for search and rescue purposes. In an emergency situation ships contact the maritime rescue coordination centre, in general using Digital Selective Calling (DSC) (which is not part of VDES). VDES would allow the rescue coordination centre to poll ships in the vicinity to ask for their SAR capabilities in an automated manner. This service would make the coordination of SAR operations easier and faster.

- Broadcast of Maritime Safety Information (MSI) and temporary and preliminary notices to mariners. This service allows a national authority to broadcast information such as weather alerts or the coordinates of areas that are to be avoided, among others.

- Transmission of Facilitation of International Maritime Traffic (FAL) forms, which are defined by IMO. These forms sent by the ship to a national authority can be used to declare the type of cargo, crew and passengers lists, etc. This speeds up the bureaucracy that needs to be completed when entering the national waters of a country.

- Vessel Traffic Service (VTS) communication. Similarly to airplanes in controlled airspace, ships intending to
Table 1 Overview of the uses cases foreseen for VDE and the communication channel (ship to shore, ship to ship, or satellite) that is used

\begin{tabular}{|c|c|c|c|}
\hline \multirow[t]{3}{*}{ Service } & \multicolumn{3}{|c|}{ Type of communication } \\
\hline & \multicolumn{2}{|l|}{ Terrestrial } & \multirow[t]{2}{*}{ Satellite } \\
\hline & Ship to shore & Ship to ship & \\
\hline SAR comm. & $\mathrm{X}$ & & $\mathrm{X}$ \\
\hline Broadcast of MSI & $\mathrm{X}$ & & $X$ \\
\hline Transmission of FAL & $\mathrm{X}$ & & $\mathrm{X}$ \\
\hline VTS communication & $X$ & & \\
\hline $\begin{array}{l}\text { Download of updated digital } \\
\text { pub. }\end{array}$ & $\mathrm{X}$ & & $X$ \\
\hline Route exchange & & $X$ & \\
\hline
\end{tabular}

enter a harbour need to establish communication with the harbour or port authorities. This communication is used mainly to guarantee navigational safety.

- Download of updated digital publications. Among others, this includes download of the Global Maritime Distress and Safety System (GMDSS) Master Plan which contains contact data (telephone numbers, postal and e-mail addresses) of the national authorities of all countries.

- Route exchange. Using AIS ships broadcast their position, course and speed to avoid collision. This service represents an improvement of AIS since it allows ships to broadcast not just their position but their intended route for the next $30 \mathrm{~min}$. This service can be of critical importance for large vessels whose course takes minutes to change due to their inertia, possibly helping in avoiding vessels collisions.

- Finally, maritime infrastructure (buoys, etc.) can broadcast information towards passing ships as an additional information source.

Table 1 compares the different use cases according to the communication channel required: ship to shore, ship to ship or satellite. We can observe how most use cases use either a direct ship to shore communication link or a satellite link. An exception is VTS communication which takes place usually only through a direct ship to shore communication, since it is only used by ship in the immediate vicinity of a harbor, where a direct ship to shore communication should be available. Finally, the only service which makes use of ship to ship communication is route exchange. Again, it only makes sense to exchange the route with vessels in the immediate vicinity which are reachable through a terrestrial ship to ship link (satellite communication does not make sense in this case).

Note that although the set of services that are originally foreseen for VDE is limited, other services might be supported in the future. 


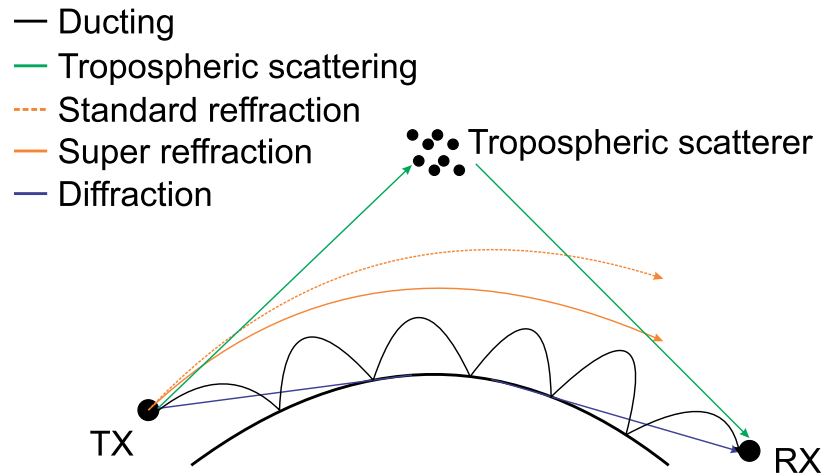

Fig. 4 Visualization of different propagation phenomena in the VHF band in the maritime environment

\section{VDE technical aspects}

\subsection{VHF wireless propagation channel}

The investigation of VHF propagation channel for the maritime environment has been mostly conducted by terrestrial based terminals. The most commonly studied propagation characteristic is the empirical path loss in different environments and under different weather conditions [10, 11]. All these models are based on numerous measurement datasets covering different scenarios. It is reported that the height of the antenna, sea condition, temperature and humidity have influence on the received power. More thorough investigations have been performed to gain insight into different propagation phenomena in sea environment [12] to derive a more precise channel model. The main focus was on evaluating individual radio paths over the sea, including the direction propagating along the LoS path, multipath propagation caused by the rough sea surface (both coherent and incoherent components), refracted path due to the change in the refractive index of the atmosphere, and diffraction caused by the objects that are between transmitter and receiver. Figure 4 visualizes the numerous effects. Further, during summer conditions the super-refraction and ducting may significantly influence the signal fading depth. In the VHF band, the signal is capable of propagating over a long distance even when the direct LoS path is blocked by the earth surface. Beyond the horizon the diffraction effect weakens and the troposcatter effect strengthens and make the VHF band an attractive frequency band for long-range communications.

The communication range depends on the height of the antennas. It can reach up to several hundreds of kilometers if line of sight prevails. Between ships with antenna heights of $30 \mathrm{~m}$ the expected range is below $40 \mathrm{~km}$ [5]. If a directional antenna is used on the shore side, the ship-shore propagation channel is similar to ship-ship channel. The main multipath is originated by the rough sea surface. If an omnidirectional antenna is used on the shore side, the multipath components originated from the land objects should be also considered. In satellite-ship channel the diffuse scattering of the microwave on the sea surface is a main source of multipath propagation.

\subsection{Modulation and coding}

One of the key features of VDE and ASM is the support of Adaptive Coding and Modulation (ACM), a technique which consists of choosing the coding rate and modulation according to the experienced Signal-to-Noise ratio (SNR), in a dynamic manner. For example, when a ship is far away from the coast, due to propagation loss, it will receive a weak signal from a coastal station, that may only be slightly stronger than the background thermal noise, i.e., it will experience a low SNR. In this case, a very robust modulation and coding scheme is used for communication, for example, $\pi / 4$ QPSK modulation with a rate $1 / 2$ channel code. This will ensure that no (or very few) packets are lost, but the obtained throughput will be low. On the other hand, as the ship gets closer and closer to the shore, its experienced SNR will become higher, allowing the use of a less robust modulation and coding scheme which, in turn, provides a higher throughput. For example, when the ship is close to the shore it might use 16-QAM modulation with a rate $3 / 4$ channel code. Both VDE and ASM support $\pi / 4$ QPSK, 8-PSK and 16-QAM modulation, and turbo codes of rate $1 / 2$ and $3 / 4$ [13].

To enable ACM, terminals and/or shore stations report the SNR they measure, which necessarily requires bidirectional communication. Thus, when a shore station communicates with a ship, the ship measures the SNR of the received signal. Shortly afterwards, when the ship communicates with the shore station, it appends its SNR measurement to the data. The next time the shore station sends data to the ship, it will choose a modulation and coding scheme according to the SNR that the ship measured and reported.

The other key component of ACM is a way of identifying the modulation. Since it is the transmitter the one who decides which modulation and coding to use, the receiver does not know which modulation and coding are used for the transmission. This problem is solved by adding a field in the packet header, which tells the receiver which modulation and coding scheme is used to transmit the remaining of the packet. In VDE and ASM, this field is encoded using a $(32,6)$ first order Reed-Muller code and modulated using QPSK modulation. This code was designed to be more robust than the most robust coding and modulation scheme that can be used for the data part of the packets. Hence, whenever communication is possible, the Reed-Muller code can be decoded with a very low error probability. 
Received samples in the decoding window

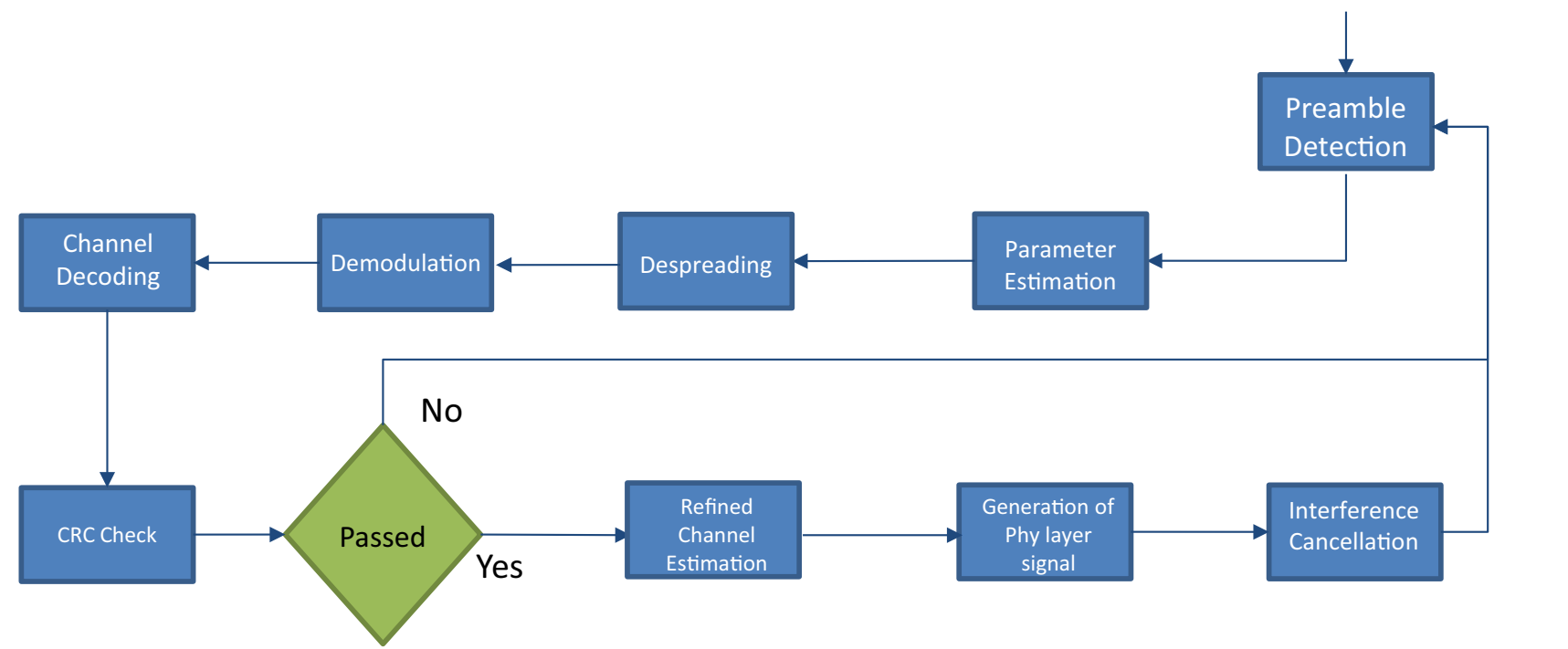

Fig. 5 The block diagram of a possible E-SSA receiver

To detect incoming packets, the terrestrial link will use a modified Barker sequence which is currently under evaluation also for the satellite link. Nonetheless, a faster variation in frequency and amplitude is expected for the satellite wireless channel compared to the terrestrial one, which will require the insertion of pilot symbols in the waveform for a suitable tracking. However, to simplify the modem, the satellite waveform is expected to be as close as possible to the terrestrial one.

\subsection{MAC protocols}

Compared to the service area of a VDE shore station, the service area of VDE satellite is much larger. This represents an advantage in many aspects, since large areas can be covered by a single satellite, but it also brings several challenges with it. The first is that a single satellite will have to serve a large number of vessels, which can become a problem in the satellite return (ship to satellite) link, since many vessels might be contending for the access to the communication channel. Hence, a high efficiency random access protocol is needed in the satellite return link. The second challenge is that ensuring that the packets from different terminals are time aligned (slot synchronous) at the satellite is challenging because of two reasons. The first effect has to do with geometry, vessels in the center of the coverage area (right below the satellite), and vessels at the edge of the coverage area will have rather different propagation delays to the satellite. The second reason is that the geometry itself changes fast due to the high speed with which satellite orbits the earth (in the order of $7.6 \mathrm{~km} / \mathrm{s}$ ). Thus, to have terminals slot aligned at the satellite one should track the satellite position and have a reliable common clock on all vessels, which would result in increased terminal complexity. Hence, to keep terminal complexity low, an asynchronous random access is preferable.

At this stage of the standardization process the best suitable candidate protocol to be used in the satellite uplink of VDE is Enhanced Spread Spectrum Aloha (E-SSA) [14]. In E-SSA, the transmitters operate like in an (asynchronous) Aloha system but use direct sequence spread spectrum [15]. The main characteristic of E-SSA is that the receiver applies successive interference cancellation (SIC). More precisely, in E-SSA a window-based decoder is normally employed. The window decoder starts storing the first $M$ samples in memory, and tries to detect the start of packets within the window. For doing so, it exploits the presence of a common preamble, which precedes the start of each packet and is known to the receiver. Once the presence of a packet is detected, the receiver attempts to decode the packet. If the packet is successfully decoded, which is verified using a Cyclic Redundancy Check (CRC), SIC is performed, i.e., its contribution to the received waveform stored in memory is canceled. This process is then repeated iteratively until all packets have been decoded, or until a maximum number of iterations have been carried out. A detailed block diagram of the E-SSA window decoder is depicted in Fig. 5.

The MAC protocols employed for ship to ship communication are rather different from E-SSA. At the MAC layer, AIS and ASM are allowed to use one out of four possible access schemes. The most widely adopted, in terms of percentage of time, or percentage of vessels, is the self-organized time division multiple access (SOTDMA) because it 
has to be used while vessels travel along a determined route. SOTDMA is based on a distributed Time Division Multiple Access (TDMA) concept, in which vessels within each others coverage range, distributively coordinate so to avoid collisions among packets. In particular, each vessels AIS receiver keeps track of the slot occupation with the help of a dynamic directory of received neighbors, and sends its messages only over slots that are perceived as free. Once a slot is occupied by a ship, it may be booked for a certain number of consecutive frames. This information is signaled in the time out field of the AIS or ASM message, so to ease negotiation procedures. Note that employing such a scheme for a satellite link is not feasible because the ships in the coverage area of the satellite cannot hear each other's transmissions.

As per the terrestrial ship-to-base-station link, TDMA is foreseen to be used in VDE. The coastal base station, upon identification of the vessels in its proximity, defines and broadcast the slot allocation plan. Hence, each vessel knows which time slots are allocated for his data transmission. No concurrent transmission are allowed, so that no interference is caused by neighboring vessels. This comes at the expense of lower degree of flexibility.

\section{Standardization process and political challenges}

Working group 3 of IALA develops a potential standard that is submitted to the working party $5 \mathrm{~B}$ of ITU for discussion. At the world radio communication conference the members of the ITU have to agree on the proposed frequencies with an expected standard in sight. The terrestrial frequencies have been allocated at the WRC-15 in 2015. The frequencies for the satellite were not confirmed yet. However, there is an agenda item for the next WRC in 2019 to discuss this. As the proposed satellite link in the downlink in this frequency band is new it may cause interference with existing landmobile systems. Therefore, it requires a cautious discussion between all member states to find an agreement at the WRC19. In Fig. 1 both currently known and discussed proposals for the satellite bands are shown. Currently it is expected that the operators of AIS satellites may upgrade their system and in addition regional operators, such as Space Norway with their scheduled Norsat-2 satellite, are interested in operating their own system.

\section{Research directions}

Despite the fact that the standardization process of VDES is quite mature and will hopefully become soon an ITU standard, there are still some research directions that can contribute to a better understanding of VDES as well as to future maritime communication systems.

The first research field is cell planning. The up and downlink channels for the terrestrial communication link are relatively narrow ( $100 \mathrm{kHz}$ in each direction). Furthermore, on sea there are hardly any obstructions to the propagation of electromagnetic waves and, as a consequence, VDES is characterized by having large communication cells. Furthermore, the cellular structure follows the shape of the shore, which as a first approximation results in a one-dimensional cell structure. An efficient cell planning is of outmost importance to utilize efficiently the scarce resources available. Furthermore, shore stations may need to cooperate with each to better use their resources in time or frequency. Furthermore, the cell planning should consider that the focus of VDES is providing coverage at large distances from the shore where no land cellular mobile systems are available.

A promising option to improve the capacity in VDES is using multiple antennas, since it is known that in principle the capacity scales linearly with the number of antennas. The frequencies used are characterized by a large wavelength (in the order of $1 \mathrm{~m}$ ), which requires not only large antennas but also a large separation between antenna elements. However, a spatial separation in the order of the meters are feasible both on the vessels as well as on the shore. Thus, we believe it is important to develop concepts with multiple antennas for VDES to improve the (cellular) capacity and to assure that VDES will not be a victim of its own success.

Despite its affordable complexity, maturity and ineluctable advantages in terms of performance, E-SSA is not the only MAC candidate option for the satellite uplink, in the VDE standard. Recently, an intense research activity is focusing on asynchronous random access [16-18] for machine-type communications and satellite communications. The key ingredients are the transmission of identical copies at physical layer with signaling information of their respective locations, coupled with SIC at the receiver, and optionally combining techniques. One of the peculiarities of the maritime uplink channel is its narrowband nature, which derives from regulatory limitations, binding the transmitters to work over a $50 \mathrm{kHz}$ band in the satellite uplink. Spread spectrum techniques are more effective when the processing gain (or spreading factor) can be driven as high as possible, ${ }^{1}$ but on the other hand, the aforementioned band limitations, allow spreading factors up to 16 only. In such scenarios, it can be shown that narrowband solutions with multiple replicas and SIC are able to outperform E-SSA. Nonetheless, practical implementations are still missing for the former solutions, while E-SSA has been shown to be practically

\footnotetext{
${ }_{1}^{1}$ It is not uncommon to see systems operating with spreading factors
} of 512 or even higher. 
viable and has been successfully adopted in standards like S-MIM [19]. Thus, further research in random access protocols could have an impact in the future of VDES.

Another field which could contribute to VDES in the future is cryptography, in particular authentication. So far, no authentication mechanisms are present in VDES, in other words, no mechanism exists to verify that a message in the VDES system was sent by a legitimate user. For example, spoofing attacks have been demonstrated in AIS. These attacks consist of creating spoofed packets and transmitting them in an AIS channel. Since no means of ensuring authenticity are available, these spoofed packets are treated as legitimate AIS packets. This could cause a legitimate AIS user to change course to avoid collision with a non-existent ship, or to join a non-existent search and rescue operation. VDES could greatly profit from authentication mechanisms, such us digital signature schemes, that could allow to discard spoofed messages.

Finally, we would like to stress that, very likely, the most promising field of research related to VDES does not have to do with the underlying technology, but more with possible applications that can be supported by VDES. As it is usually the case in digital communications, once communication is made possible with a good quality of service, new applications start appearing bringing far reaching benefits which nobody could have never conceived.

\section{Conclusions}

The maritime community is seeking for new possibilities and capacities towards digital communications. Since the users are on globally distributed ships and often time far beyond the horizon, a hybrid solution of a terrestrial and satellite-based transmission scheme addresses the challenge for reaching all users. The VDES is currently in the final standardization process including a digital communications component for any kind of communication, namely VDE. This Article shows that at the beginning a full understanding of the physical behavior in the VHF band is needed. This relates to the propagation characteristics of all envisaged links (terrestrial up- and downlinks, as well as the satellite up- and downlinks). Then, an appropriate signal transmission scheme can be designed including the task of minimal interference between the terrestrial and satellite link. This includes all different coding and modulation schemes, also the finding of the best suited access scheme. Hereby, Enhanced Spread Spectrum Aloha (E-SSA) is proposed. Future fields of research are identified, e.g., cellular design, multiple antenna schemes. Finally, all the diverse user requirements, use cases, regulatory restrictions have to be taken into account, before a final VDE standard is alive.
Open Access This article is distributed under the terms of the Creative Commons Attribution 4.0 International License (http://creativeco mmons.org/licenses/by/4.0/), which permits unrestricted use, distribution, and reproduction in any medium, provided you give appropriate credit to the original author(s) and the source, provide a link to the Creative Commons license, and indicate if changes were made.

\section{References}

1. Recommendation ITU-R M.1371-0: Technical characteristics for a universal shipborne automatic identification system using time division multiple access in the VHF maritime mobile band. ITU, Technical Report (1998)

2. Recommendation ITU-R M.1371-5: Technical characteristics for an automatic identification system using time division multiple access in the VHF maritime mobile frequency band. ITU, Technical Report (2014)

3. IMO SN.1/Circ.289: Guidance on the Use of AIS ApplicationSpecific Messages. IMO (2010)

4. Recommendation ITU-R M.1371-2: Technical characteristics for an automatic identification system using time division multiple access in the VHF maritime mobile band. ITU, Technical Report (2007)

5. Griffiths, H.D., Vinagre, L., Vines, S.B., Bartram, C.P.: Measurements of non line-of-sight VHF propagation over the sea surface. In: Tenth International Conference on Antennas and Propagation (Conference Publication No. 436), vol. 2, pp. 278-280 (1997)

6. Recommendation ITU-R M.2371: Selection of the channel plan for a VHF data exchange system. ITU, Technical Report (2009)

7. Resolution 360 (REV.WRC-15): In: World Radiocommunication Conference, Geneva (2015)

8. Clazzer, F., Lázaro, F., Plass, S.: Enhanced AIS receiver design for satellite reception. CEAS Space J. 8(4), 257-268 (2016)

9. Plass, S., Poehlmann, R., Hermenier, R., Dammann, A.: Global maritime surveillance by airliner-based AIS detection: preliminary analysis. J. Navig. 68, 1195-1209 (2015)

10. Timmins, I.J., O'Young, S.: Marine communications channel modeling using the finite-difference time domain method. In: IEEE Trans. Veh. Technol. 58(6), 2626-2637 (2009)

11. Recommendation ITU-R P.1546-5: Method for point-to-area predictions for terrestrial services in the frequency range $30 \mathrm{MHz}$ to $3000 \mathrm{MHz}$, P Series (2013)

12. Sim, C.Y.D.: The propagation of VHF and UHF radio waves over sea paths. Ph.D. Thesis (2002)

13. Recommendation ITU-R M.2092: Technical characteristics for a VHF data exchange system in the VHF maritime mobile band. ITU, Technical Report, 2017, Working Version of IALA WG3

14. del Rio Herrero, O., De Gaudenzi, R.: High efficiency satellite multiple access scheme for machine-to-machine communications. IEEE Trans. Aerosp. Electron. Syst. 48(4), 2961-2989 (2012)

15. Pickholtz, R.L., Schilling, D.L., Milstein, L.B.: Theory of spreadspectrum communications-a tutorial. IEEE Trans. Commun. 30(5), 855-884 (1982)

16. Kissling, C.: Performance enhacements for asynchronous random access protocols over satellie. In: IEEE ICC 2011. Kyoto, Japan (2011)

17. De Gaudenzi, R., del Rio Herrero, O., Acar, G., Garrido Barrabes, E.: Asynchronous contention resolution diversity ALOHA: making CRDSA truly asynchronous. IEEE Trans. Wireless Commun. 13(11), 6193-6206 (2014)

18. Clazzer, F., Kissling, C.: Enhanced Contention Resolution ALOHA-ECRA. In: International ITG Conference on Systems, Communications and Coding, Munich, Germany (2013) 
19. Scalise, S., Párraga Niebla, C., De Gaudenzi, R., del Rio Herrero, O., Finocchiaro, D., Arcidiacono, A.: S-MIM: a novel radio interface for efficient messaging services over satellite. IEEE Commun. Mag. 51(3), 119-125 (2013) 